\title{
Laser cirúrgico no tratamento de hiperplasia fibrosa
}

Surgical laser on fibrous hyperplasia treatment

\author{
Alessandra Kuhn-Dall'Magro* \\ Jonathan Rodrigo Lauxen** \\ Renato dos Santos ${ }^{* * *}$ \\ Roberta Neuwald Pauletti*** \\ Eduardo Dall'Magro ${ }^{* * * *}$
}

\section{Resumo}

Objetivo: demonstrar, por meio de relato de caso clínico, a exérese de uma hiperplasia fibrosa inflamatória (HFI) com o uso de laser cirúrgico de diodo. Relato de caso: paciente leucoderma, gênero masculino, 48 anos, compareceu ao Ambulatório de Especialidades do SUS do Hospital São Vicente de Paulo, Passo Fundo/RS, apresentando ausência do elemento dentário 46 e lesão nodular exofítica, pediculada, coloração rósea, superfície lisa em mucosa jugal medindo aproximadamente $2 \mathrm{~cm}$, ocupando o espaço do elemento ausente, com histórico de 6 anos, sem queixa álgica no local. $O$ tratamento proposto foi a remoção cirúrgica com laser de diodo, seguindo protocolo sugerido pelo fabricante. Sob anestesia infiltrativa local, realizou-se a excisão da lesão com incisão uniforme pela base. A peça cirúrgica foi fixada em formol $10 \%$ e encaminhada para exame anatomopatológico. O exame histopatológico apresentou como diagnóstico fibroma de irritação. Considerações finais: o uso de laser cirúrgico de diodo é um método seguro e eficaz, além de apresentar inúmeras vantagens quando comparado com a técnica convencional para excisões cirúrgicas de lesões de tecidos moles na cavidade oral. A remoção cirúrgica da HFI com pequena margem de segurança e remoção do fator etiológico constitui uma terapêutica satisfatória; a amostra deve sempre ser enviada para análise anatomopatológica, para confirmar o diagnóstico. O cirurgião-dentista deve ter conhecimento específico para operação e indicação do uso do laser cirúrgico.

Palavras-chave: Hiperplasia fibrosa. Patologia bucal. Cirurgia bucal. Laser.

\section{Introdução}

A hiperplasia fibrosa inflamatória (HFI) ou hiperplasia fibrosa traumática (HFT) é uma lesão benigna, proveniente de reação hiperplásica do tecido conjuntivo fibroso em resposta a injúrias crônicas de baixa intensidade ${ }^{1}$. O aparecimento da lesão está frequentemente relacionado com o uso de próteses dentárias mal adaptadas ou com câmara de sucção $0^{1-3}$. Pode-se citar, também, como fatores etiológicos, diastemas, bordos cortantes de dentes, procedimentos iatrogênicos e higiene bucal inadequada ${ }^{1,4}$.

Clinicamente, a lesão apresenta-se como massa tumoral de tecido conjuntivo fibroso, consistência flácida ou firme, de base séssil ou pediculada, superfície lisa ou vegetante, semelhante à hiperplasia papilar, coloração semelhante à mucosa ou eritematosa, crescimento lento e geralmente assintomáti$\mathrm{ca}^{1,2,4,5}$

As características histológicas mais comuns são epitélio pavimentoso estratificado, queratinizado ou não, que envolve tecido conjuntivo fibroso hiperplásico, com número elevado de fibras colágenas, apresentando, também, alto grau de células inflamatórias crônicas e quantidade variável de vasos sanguíneos ${ }^{1,2,5}$.

Especialista em CTBMF, Santa Casa, Porto Alegre; mestre em Ciências Médicas - UFRGS; professora dos cursos de pós-graduação em CTBMF do Centro de Estudos Odontológicos Meridional (CEOM), Passo Fundo e do Instituto Odontológico das Américas (IOA), Balneário Camboriú, SC; membro do corpo clínico do Hospital São Vicente de Paulo de Passo Fundo (HSVP) e do Instituto de Ortopedia e Traumatologia (IOT), Passo Fundo, RS, Brasil.

Cirurgião-dentista graduado pela Faculdade de Odontologia da Universidade de Passo Fundo (UPF), Passo Fundo, RS, Brasil.

Aluno do curso de Especialização em CTBMF-CEOM-HSVP, Passo Fundo - RS, Brasil.

Especialista em CTBMF pela UPF-HSVP; professora dos cursos de pós-graduação em CTBMF do CEOM, Passo Fundo, RS, Brasil.

Especialista em Prótese Dentária pela USP, mestre em Laser em Odontologia (Lelo) pela USP, mestre e doutor em Materiais Dentários pela Unicamp, professor titular III da Faculdade de Odontologia da UPF, Passo Fundo, RS, Brasil. 
O tratamento para a HFI é a remoção cirúrgica (com bisturi convencional, elétrico ou laser cirúrgico) com pequena margem de segurança seguido da remoção do agente causal. Outras formas terapêuticas são microabrasão e crioterapia ${ }^{1,5,6}$.

O exame histopatológico é importante para confirmação do diagnóstico clínico, já que a HFI faz diagnóstico diferencial com lipofibroma, neurofibroma, rabdomioma, leiomioma, tumores de glândulas salivares menores, granuloma piogênico e fibroma ossificante periférico ${ }^{1,6,7}$.

O objetivo do presente trabalho é demonstrar, por meio de relato de caso clínico, a exérese de uma HFI com o uso de laser cirúrgico de diodo.

\section{Revisão de literatura}

Os lasers para tecido mole são utilizados em incisões e hemostasias, pois são bem absorvidos por pigmentos e sangue. A sua aplicação resulta em três zonas sobre o tecido hígido: vaporização, necrose e coagulação. Essas zonas variam de tamanho de acordo com a energia e o foco de aplicação. Quanto maior a energia, maior a zona de vaporização e, consequentemente, maior a profundidade da incisão, com a vantagem de ser livre de sangramento e reduzir o número de microrganismos. Quanto menor a energia, menor é a vaporização e maior a zona de coagulação. Quanto mais focado, menor é o diâmetro e maior a profundidade; por fim, quanto mais desfocado, maior é o efeito de coagulação e hemostasia $^{5,8,9-11}$.

Diversos autores ${ }^{5,8,9-13}$ destacam as vantagens do uso de laser de alta potência para cirurgia de tecidos moles. Dentre elas, pode-se citar: hemostasia, redução da dor e infecção pós-operatória, menor contração tecidual, eliminação da necessidade de sutura, menor tempo cirúrgico, redução do trauma, edema e cicatrizes. Em lesões malignas ou cancerizáveis, existe menor risco de metástase, uma vez que o laser faz o selamento imediato dos vasos sanguíneos e linfáticos.

Segundo Pedron et al. ${ }^{5}$, foi relatada redução da necessidade do uso de anestesia em alguns procedimentos, como incisão e drenagem de abscessos, ulectomia, frenectomia, anquilotomia, gengivectomia, gengivoplastia, curetagem de bolsa periodontal e excisão de tumores pediculados. Ainda, conforme os autores, a redução de formação de cicatrizes pode ser explicada pela matriz extracelular remanescente, que não é destruída pelo laser, funciona como um splint mecânico que impede o tecido de granulação de contrair-se. As proteínas extracelulares residuais também são iguais para a neoformação de tecido epitelial (migração epitelial guiada), pois se interpõem entre os fibroblastos, impedindo maior aproximação entre eles e prevenindo, simultaneamente, a formação de filamentos contrácteis, capazes de distorcer os tecidos em cicatrização. Dessa forma, a "desvantagem" da reparação retardada torna-se vantajosa, por haver tempo hábil para as células formarem um arcabouço extracelular hígido que não pode ser destruído por forças celulares fracas.

\section{Relato de caso}

Paciente leucoderma, masculino, 48 anos, procurou atendimento odontológico apresentando lesão nodular em mucosa jugal. Na anamnese, relatou ter percebido o aparecimento da lesão há cerca de 6 anos, não referindo dor no local. No exame clínico intraoral, percebeu-se ausência do elemento dentário 46 e lesão exofítica, pediculada, coloração rósea, superfície lisa e mucosa jugal com aproximadamente $2 \mathrm{~cm}$ de diâmetro, ocupando o espaço do elemento ausente (Figura 1).

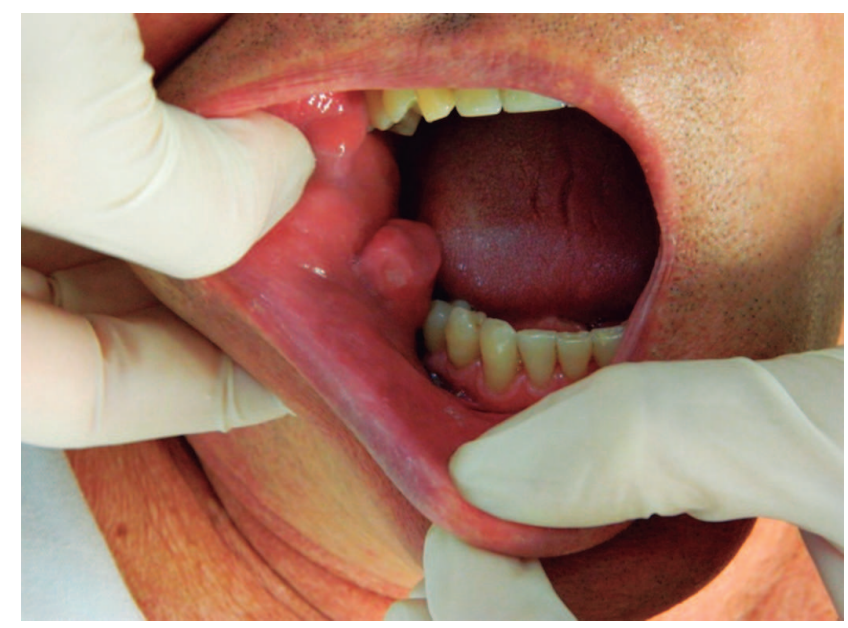

Figura 1 - Aspecto clínico inicial da lesão

Pelas características clínicas e pelo histórico da lesão, foi sugerido diagnóstico clínico de hiperplasia fibrosa inflamatória.

O tratamento proposto foi a remoção cirúrgica com laser de diodo Thera Lase Surgery (DMC Equipamentos, São Carlos/SP, Brasil) com comprimento de onda de $808 \mathrm{~nm}$ e meio ativo de Gallium-Indium Arsenide (InGaAs), seguindo protocolo sugerido pelo fabricante (Figura 2 - A).

Sob anestesia infiltrativa local, realizou-se a excisão da lesão com potência de $4400 \mathrm{~mW}$, energia de $4 \mathrm{~J}$ e frequência contínua. A lesão foi completamente removida com incisão uniforme pela base (Figura $2-\mathrm{B}$ e C). 


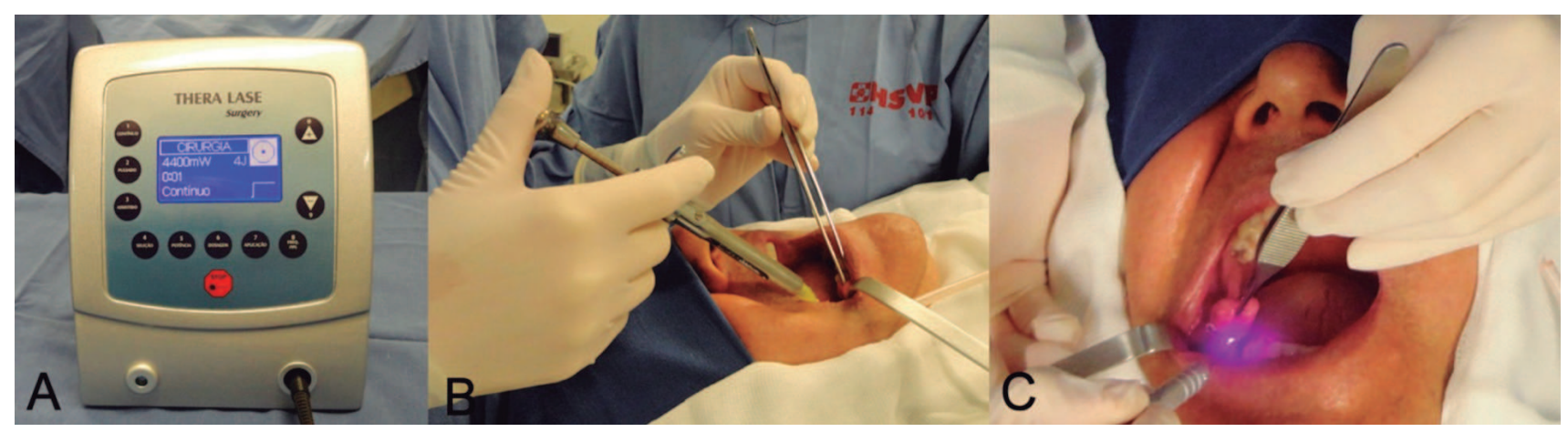

Figura 2 - A: Thera Lase Surgery (DMC Equipamentos, São Carlos/SP, Brasil); B: anestesia infiltrativa; C: incisão com laser de diodo

A peça cirúrgica consistia em um fragmento ovalado de mucosa jugal, medindo $1,7 \times 1,2 \times$ $1,0 \mathrm{~cm}$, apresentando na superfície lesão nodular branco-acinzentada (Figura 3-A). Foi fixada em formol $10 \%$ e encaminhada para exame anatomopatológico. $\mathrm{O}$ exame evidenciou a presença de fibroma de irritação (Figura 3 - B e C).

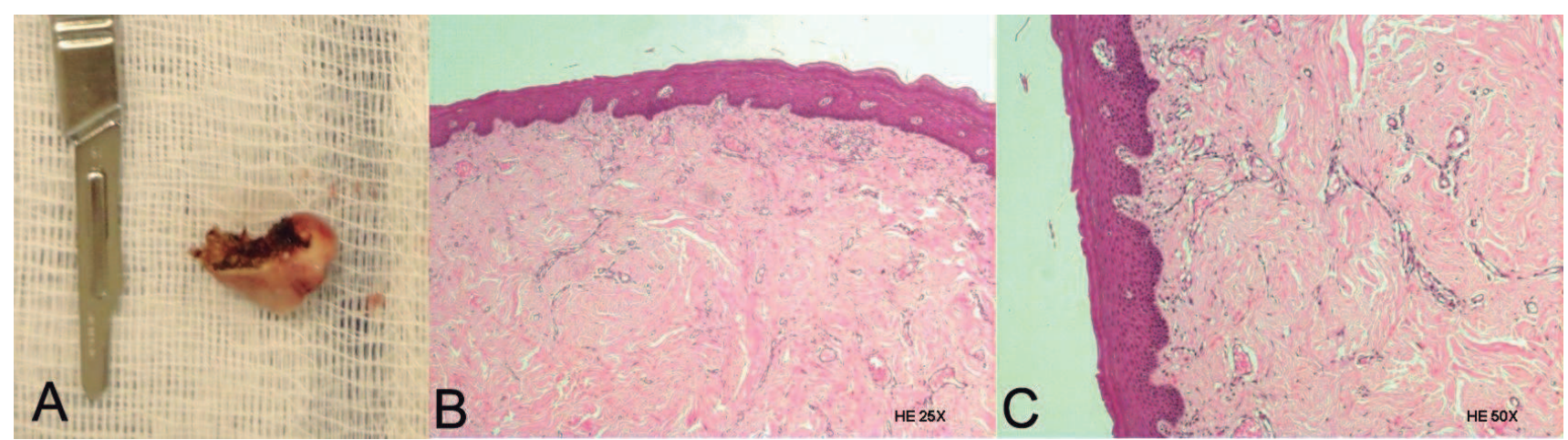

Figura 3 - A: peça cirúrgica; B: HE 25X; C HE 50X: epitélio escamoso achatado na porção superior, sob abundante colágeno vascularizado

Foram adotados cuidados pós-operatórios de rotina com colocação de gelo na face, repouso com cabeça elevada, bochechos com clorexedina a $0,12 \%$ para higiene oral, antibiótico, anti-inflamatório e analgésico EV por 24 horas, recebendo alta após esse período. No pós-operatório, o paciente não relatou algia no local do procedimento (Figura $4-\mathrm{A}$, $\mathrm{B}$ e C).

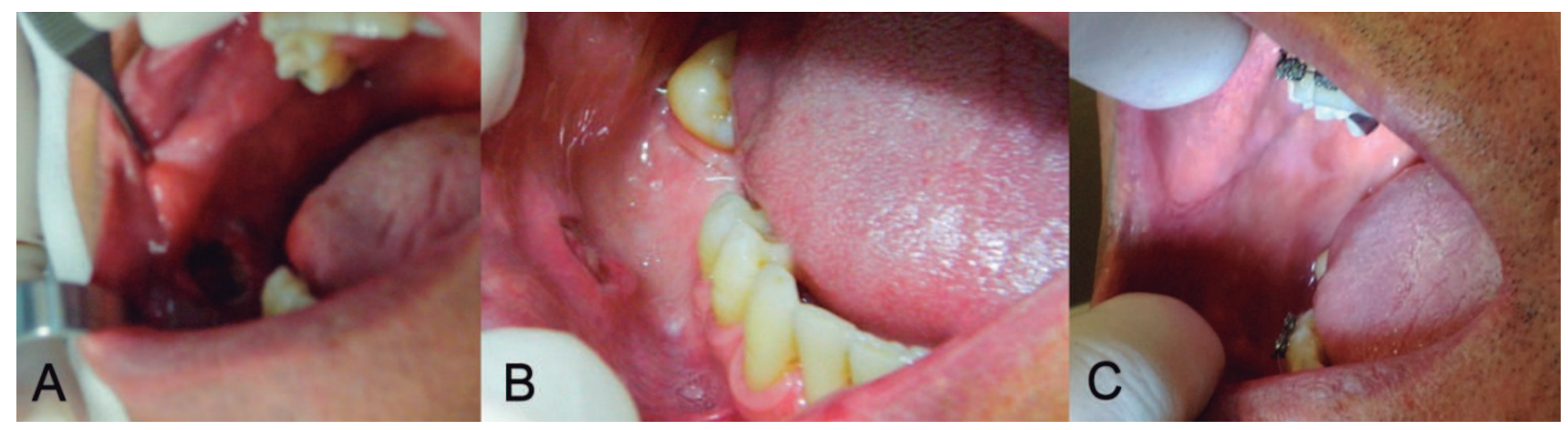

Figura 4 - A: pós-operatório imediato; B: pós-operatório de 15 dias; C: pós-operatório de 90 dias

\section{Discussão}

O laser tem sido cada vez mais utilizado nas mais diversas áreas da saúde ${ }^{13}$. Na odontologia, pode ser usado em procedimentos em tecidos moles e duros, tais como gengivectomias, ulotomias, ulectomias, aumento de coroa clínica, acesso aos implantes, incisão para drenagem de abcessos bucais, excisão de diversos tumores, hemangiomas e outras lesões vasculares, cistos de erupção, tratamento de bolsa periodontal, remoção de cárie dentária, selamento de cicatrículas e fissuras, entre outros ${ }^{5,8,9-11}$.

Em cirurgias de tecido mole, o uso do laser apresenta diversas vantagens, tais como hemostasia, redução da dor pós-operatória, diminuição do uso de anestésicos locais, além de outras já citadas. 
Em estudo comparativo entre cirurgia convencional e laser de $\mathrm{CO}_{2}$, para remoção de fenômeno de retenção de muco (Mucocele), Fernandes et al. ${ }^{14}$ compararam o transoperatório e o pós-operatório em cada uma das técnicas utilizadas. No grupo da técnica convencional (nove pacientes) houve episódios hemorrágicos no transcirúrgico. No pós-operatório, oito pacientes relataram dor, principalmente nas primeiras 24 horas, sendo necessário o uso de analgésicos; além disso, todos os pacientes apresentaram edema. Já no grupo que utilizou o laser (15 pacientes), apenas três relataram dor, porém sem a necessidade do uso de analgésicos. A cicatrização foi melhor no grupo do laser, tendo aspecto final mais estético, uma vez que, no grupo da técnica convencional, ocorreram casos de formação de queloides.

Kondori et al. ${ }^{15}$ analisaram 976 biopsias e compararam a hipótese diagnóstica clínica com o diagnóstico final, após exame histológico. Em $43 \%$ dos casos, houve erro de diagnóstico, tendo a HFI sido diagnosticada incorretamente em $10 \%$. Portanto, os autores recomendam que todas as lesões excisadas devem ser submetidas a exame histopatológico.

Vescovi et al. ${ }^{16}$ estudaram os efeitos do uso de laser de Nd:YAG no diagnóstico histológico. Foram submetidas a remoção cirúrgica e análise histológica 26 lesões, das quais 15 foram removidas com o uso do laser e 11, excisionadas com lâmina de bisturi. Os autores concluíram que não há interferência no resultado do exame histológico, independentemente da técnica utilizada, desde que a lesão seja maior que $7 \mathrm{~mm}$. Já para Angiero et al. ${ }^{17}$, que também estudaram os danos causados pelo uso do laser na remoção de lesões em tecido mole e a sua interferência na análise histológica, a lesão deve ter no mínimo $5 \mathrm{~mm}$ para que os danos não interfiram nesse tipo de diagnóstico.

Em estudo para avaliar a segurança e eficácia do uso do laser de diodo em cirurgias orais, Desiate et al. ${ }^{18}$ submeteram cinco pacientes à cirurgia de freio labial. Não houve necessidade do uso de anestesia local e, durante o procedimento, não houve ocorrência de hemorragia. Os pacientes descreveram o procedimento como indolor, não necessitando de sutura, e em 10 dias a ferida cirúrgica estava cicatrizada.

No presente caso clínico, assim como na literatura consultada, foi possível observar as vantagens do uso do laser cirúrgico, em comparação com a técnica convencional para excisão de tecido mole.

\section{Considerações finais}

Tendo como base a literatura revisada e o estudo do caso clínico, é possível concluir que:

- O uso de laser cirúrgico de diodo é um método seguro e eficaz, além de apresentar inúmeras vantagens, quando comparado com a técnica convencional, para excisões cirúrgicas de lesões de tecidos moles na cavidade oral.

- A remoção cirúrgica da HFI com pequena margem de segurança e remoção do fator etiológico constitui uma terapêutica satisfatória.

- A amostra deve sempre ser enviada para análise anatomopatológica, para confirmar o diagnóstico.

- O cirurgião-dentista deve ter conhecimento específico para operação e indicação do uso do laser cirúrgico.

\section{Abstract}

Objective: to demonstrate through a clinical case report the excision of an inflammatory fibrous hyperplasia (IFH) with the use of surgical diode laser. Case report: male leucoderma patient, 48 years old, sought assistance on the Specialty Outpatient Clinic of SUS (Unified Health System) of the Hospital São Vicente de Paulo, in the city of Passo Fundo / RS - Brazil, lacking tooth 46 and with nodular exophytic lesion, pedunculated, with a pinkish color and smooth surface, on the buccal mucosa measuring approximately $2 \mathrm{~cm}$ and occupying the space of the missing element. The patient had a history of 6 years without pain complaint on site. The proposed treatment was surgical removal with diode laser, following manufacturer's suggested protocol. Under local infiltrative anesthesia, an excision of the lesion with uniform incision at the base was made. The surgical specimen was fixed in 10\% formaldehyde and sent for pathological examination. Histopathological examination presented fibroma irritation as diagnosis. Final considerations: the use of surgical diode laser is a safe and effective method, and presents numerous advantages compared to the conventional technique for surgical excisions of soft tissue lesions in the oral cavity. Surgical removal of IFH with small safety margin, and removal of the etiologic factor is a satisfactory therapy. The sample should always be sent for pathological examination to confirm the diagnosis. The dentist must have specific knowledge to operate and recommend the use of surgical laser.

Keywords: Fibrous hyperplasia. Oral pathology. Oral surgery. Laser. 


\section{Referências}

1. Falcão AFP, Lamberti PLR, Lorens FGL, de Lacerda JA, Nascimento BC. Hiperplasia fibrosa inflamatória: relato de caso e revisão de literatura. Rev Ciên Med Biol 2009; 8(2):230-6.

2. Neville BW, Allen CM, Damm DD, Bouquot JE. Patologia oral e maxilofacial. 2. ed. Rio de Janeiro: Guanabara Koogan; 2004.

3. De Carli JP, Bernabé DG, Kawata L, Moraes NP, da Silva SO. Hiperplasia palatina por câmara de sucção tratada por meio da eletrocirurgia: relato de dois casos clínicos. Rev Fac Odontol UPF 2006; 11(2):81-5.

4. Paraguassú GM, Pimentel PA, Santos AR, Gurgel CAS, Sarmento VA. Prevalência de lesões bucais associadas ao uso de próteses dentárias removíveis em um serviço de estomatologia. Rev Cub Estomatol 2011; 48(3):268-76.

5. Pedron IG, Carnava TG, Utumi ER, Moreira LA, Jorge WA. Hiperplasia fibrosa causada por prótese: remoção cirúrgica com laser Nd:YAP. Rev Clin Pesq Odontol 2007; 3(1):51-6.

6. Santos MESM, Costa WRM, da Silva Neto JC. Terapêutica cirúrgica da hiperplasia fibrosa inflamatória - relato de caso. Rev Cirurg Traumatol Buco-Maxilo-Facial 2004; 4(4):241-5.

7. Vaz DA, Valença DL, Lopes RBM, Silva AVC, Pereira JRD. Concordância entre os diagnósticos clínicos e histopatológicos do Laboratório de Patologia Bucal da Faculdade de Odontologia de Pernambuco. Rev Pós Grad 2011; 18(4):23643.

8. Santos ESR, Imparato JCP, Adde CA, Moreira LA, Pedron IG. Frenectomia a laser (Nd:YAP) em Odontopediatria. Rev Odonto 2007; 15(29):107-13.

9. Jorge ACT, Cassoni A, Rodrigues JÁ. Aplicações dos lasers de alta potência em odontologia. Rev Saúde 2010; 4(3):25-33.

10. Kreisner PE, Viegas VN, Pereira ACL, Pagnoncelli RM. Excisão cirúrgica de hemangioma labial com laser de Nd:YAG. Rev Fac Odonto 2004; 45(2):39-42.

11. Johar K. Fundamentals of laser dentistry. New Delhi: Jaypee Brothers Medical Publishers; 2011.

12. Raldi FV, Niccoli-Filho W, Prakki P. Estudo clínico comparativo entre reparo após sutura convencional e incisão a laser de dióxido de carbono $\left(\mathrm{CO}_{2}\right)$ com diferentes potências. Pós Grad Rev Fac Odonto 1999; 2(1):29-35.

13. Kuhn A, Dall'Magro E, Rhoden RM. Visão multidisciplinar dos lasers de Érbio (Er:YAG) e dióxido de carbono $\left(\mathrm{CO}_{2}\right)$. Rev Médica HSVP 2001; 13(29):22-4.

14. Fernandes AMM, Morosolli ARC, Farias LP, Niccoli-Filho W, Estudo clínico comparativo entre cirurgia convencional e radiação laser de $\mathrm{CO}_{2}$ em fenômeno de retenção de muco. Cinc Odontol Bras 2006; 9(1):54-61.

15. Kondori I, Mottin RW, Laskin DM. Accuracy of dentists in clinical diagnosis of oral lesions. Quintessence Int 2011; 42(9):557-75.

16. Vescovi P, Corcione L, Meleti M, Merigo E, Fornaini C, Manfredi M, et al. Nd:YAG laser versus traditional scalpel. A preliminary histological analysis of specimens from the human oral mucosa. Lasers Med Sci 2010; 25(5):685-91.

17. Angiero F, Parma L, Crippa R, Benedicenti S. Diode laser $(808 \mathrm{~nm})$ applied to oral soft tissue lesions: a retrospective study to assess histopathological diagnosis and evaluate physical damage. Lasers Med Sci 2012; 27(2):383-8.
18. Desiate A, Cantore S, Tullo D, Profeta G, Grass FR, Ballini A. $980 \mathrm{~nm}$ Diode laser in oral and facial practice: current of the science and art. Int J Med Sci 2009; 6(6):358-64.

\section{Endereço para correspondência:}

Alessandra Kuhn-Dall'Magro

Rua Teixeira Soares, 777/ 02 e 301,

Centro 99010-080 Passo Fundo, RS

Fone: 54 3316-8402

E-mail: alessandrakuhn@hotmail.com

Recebido: 13/08/2013. Aceito: 10/09/2013. 